\title{
Reduced BMP6 expression by DNA methylation contributes to EMT and drug resistance in breast cancer cells
}

\author{
GENG LIU $^{1}$, YUAN-JIE LIU ${ }^{1}$, WEN-JING LIAN ${ }^{1}$, ZHI-WEI ZHAO ${ }^{1}$, TAO $^{2}{ }^{2}$ and HONG-YING ZHOU ${ }^{1}$ \\ ${ }^{1}$ Department of Human Anatomy, West China School of Preclinical and Forensic Medicine, Sichuan University; \\ ${ }^{2}$ Biotherapy Laboratory of Gynecological Oncology, Key Laboratory of Obstetric and Gynecologic \\ and Pediatric Diseases and Birth Defects of The Ministry of Education, West China \\ Second Hospital, Sichuan University, Chengdu, Sichuan 610041, P.R. China
}

Received February 17, 2014; Accepted April 23, 2014

DOI: $10.3892 /$ or.2014.3224

\begin{abstract}
Bone morphogenetic protein 6 (BMP6) is an important regulator of cell growth, differentiation and apoptosis in various types of tumor. In breast cancer, it was considered as a tumor suppressor. Our previous study also confirmed that BMP6 was a critical regulator of breast cancer drug resistance. However, little is known about how its expression is regulated and its mechanisms in breast cancer drug resistance. In the present study, we assessed the DNA methylation regulation of BMP6 based on the presence of a large $\mathrm{CpG}$ island in the BMP6 gene promoter. Quantitative DNA methylation analyses showed a significantly increased DNA methylation level in the drug-resistant cell line MCF-7/ADR compared to their parental cells MCF-7. Moreover, the drug-resistant cell line MCF-7/ADR showed an EMT phenotype confirmed by morphology and the expression of EMT marker gene. MCF-7 cells transfected with BMP6-specific shRNA vector also showed an EMT phenotype. The MCF-7/ADR cells treated with the recombinant BMP6 proteins reversed their EMT phenotype. These data indicated that hypermethylation modifications contributed to the regulation of BMP6 and induced an EMT phenotype of breast cancer during the acquisition of drug resistance.
\end{abstract}

\section{Introduction}

The epithelial-mesenchymal transition (EMT) is a complex biological process by which the epithelial cells go through

Correspondence to: Dr Hong-Ying Zhou, Department of Human Anatomy, West China School of Preclinical and Forensic Medicine, Sichuan University, Chengdu, Sichuan 610041, P.R. China

E-mail: eaglezhyxzy@163.com

Dr Tao Yi, Biotherapy Laboratory of Gynecological Oncology, Key Laboratory of Obstetric and Gynecologic and Pediatric Diseases and Birth Defects of the Ministry of Education, West China Second Hospital, Sichuan University, Chengdu, Sichuan 610041, P.R. China E-mail:yitao-yt@sohu.com

Key words: BMP6, breast cancer, DNA methylation, EMT, drug resistance multiple biochemical changes mainly including the loss of epithelial characteristics and acquisition of a mesenchymal motile phenotype, which enhance migratory capacity, invasiveness, elevate resistance to apoptosis and increase production of the extracellular matrix components (1-5). It has been confirmed that EMT is closely related to cancer development and progression. Increasing evidence suggests that the cancer cell acquisition of EMT phenotype is critically involved in tumor invasion and metastasis $(6,7)$. Recently, the EMT characteristics were also found in cancer cells which acquired chemoresistance after chemotherapy (8). For example, EMT is reportedly associated with the resistance to gefitinib and erlotinib in NSCLC (9-11). In breast and ovarian cancer, chemoresistance can also induce EMT via upregulation of Snail, which is a key regulator of EMT $(12,13)$. These data indicated that EMT is strongly involved in cancer cell drug resistance, but the underlying mechanisms remain unknown.

Breast cancer is the main cause of cancer-related mortality among women and drug resistance is the major clinical obstacle to the successful treatment. There are a series of molecular mechanisms which are involved in breast cancer cell drug resistance including gene mutation, gene amplification, epigenetic change and microRNAs $(14,15)$. In our previous study, we found that BMP6 played a critical role in breast cancer progression and drug resistance (16). However, during the acquisition of chemoresistance of breast cancer, the regulation of BMP6 expression and the function of BMP6 remained unknown. DNA methylation is an efficacious mechanism to regulate gene expression during development, differentiation and carcinogenesis of various tumors. DNA methylation has previously been described to act during the EMT program in the suppression of epithelial markers and the conversion of epithelial cells into aggressive, invasive tumor cells $(17,18)$.

Herein, we performed analyses to identify BMP6 which was regulated by DNA methylation and which regulated the EMT of drug-resistant cells. We found that the promoter of BMP6 is hypermethylated significantly in drug-resistant breast cancer cells that have undergone EMT. These results suggest that aberrant DNA methylation of BMP6 is critical in the drug resistance and EMT phenotype in breast cancer. 


\section{Materials and methods}

Patients and tissue specimens. A total of 16 fresh breast cancer tissues and patient-matched adjacent normal breast cancer tissues from West China Hospital of Sichuan University were used in this study. None of the patients received chemotherapy or radiotherapy prior to surgery. The study was approved by the local ethical standards of the institutional review board.

RNA isolation and quantitative RT-PCR. The expression of BMP6 was analyzed using SYBR-Green PCR master mix (Tiangen). Real-time PCR was performed on Mastercycler ep realplex (Eppendorf). Levels of mRNA expression were quantified after normalization with endogenous control GAPDH using the 2- $\Delta$ CT method (19). The primer sequences used for PCR were: GAPDH: 5'-ctttggtatcgtggaaggactc-3', 5'-gtagaggcagggatgatgttct-3' (product size, 132 bp); bmp6: 5'-gtcttacaggagcatcagcacag-3', 5'-ggagtcacaaccacagattg-3' (product size, 128 bp); ZEB1: 5'-ctgattcccaggtggcata-3', 5'-ggg cggtgtagaatcagagt-3' (product size, 168 bp); Twist1: 5'-agtggt tcttctgcgctact-3', 5'-gtagggctgctggaaggtaa-3' (product size, $161 \mathrm{bp}$ ); E-cadherin: 5'-cgtagcagtgacgaatgtgg-3', 5'-ctgggcag tgtaggatgtga-3' (product size, $175 \mathrm{bp}$ ); Vimentin: 5'-cgccaacta catcgacaagg-3', 5'-ggctttgtcgttggttagct-3' (product size, 166 bp). Experiments were performed independently for each sample and at least 3 technical replicates were run for each treated sample and controls.

Cell culture. MCF7 cell line was purchased from American Type Culture Collection (ATCC) and doxorubicin-resistant subline (MCF7/Adr) was generated by continuously culturing the drug sensitive parental cell line (MCF7) in medium containing incrementally increasing concentrations of doxorubicin (Sigma-Aldrich). The cells were cultured in RPMI-1640 (Gibco) supplemented with $10 \%$ heat inactivated fetal calf serum, $100 \mathrm{U} / \mathrm{ml}$ penicillin, and $100 \mathrm{U} / \mathrm{ml}$ streptomycin at $37^{\circ} \mathrm{C}$ and $5 \% \mathrm{CO}_{2}$. The drug-resistant cell line was cultured in drug-free medium for $>2$ weeks before subsequent experiments to avoid the influence of the drug. MCF-7/ADR were treated with $5 \mu \mathrm{M} 5$-aza-2'-deoxycytidine (5-aza-dC; Sigma-Aldrich) or $200 \mathrm{ng} / \mathrm{ml}$ rhBMP-6 (R\&D Systems) for $72 \mathrm{~h}$.

BMP6 small hairpin RNA (shRNA) transfection and isolation of clones stably expressing BMP-6 shRNA. MCF-7 cells grown on 24-well plates were transfected either with pGPU6/ GFP/NEO-BMP6 or negative control plasmid (Genepharma) using Lipofectamine 2000. After another 12-h incubation, the cells were re-plated at $1 / 10$ dilution onto the 6 -well plates. Selection with G418 $(500 \mu \mathrm{g} / \mathrm{ml})$ started on the next day and the process lasted for 2 weeks. MCF-7 cell clones with stably decreased expression of BMP6 as well as the control clones were obtained for further study. BMP6 knockdown was evaluated by real-time PCR and western blotting.

Western blot analysis. Tissue samples or cell pellets were lysed in RIPA buffer containing complete protease inhibitor cocktail (Millipore). Proteins were quantified with BCA Protein Assay (Bio-Rad) and separated by SDS-PAGE. Blots were probed using anti-BMP6 (ab155963, Abcam), anti-E-cadherin (ab53033, Abcam) and anti-Vimentin (sc-6260, Santa Cruz).
Proteins were detected using horseradish peroxidase-conjugated secondary antibodies.

Quantitative DNA methylation analyses. Genomic DNA of 16 cancers and adjacent normal colorectal tissues or cells were extracted by Tissue DNA kit (Qiagen) according to the manufacturer's instructions. A total of $200 \mathrm{ng}$ genomic DNA from each sample was bisulfite-treated with the Methylamp DNA Modification kit (Epigentek). The quality of the bisulfite conversion was controlled by using PCR products. The Sequenom MassARRAY platform was used to perform the quantitative methylation analysis of BMP6. We analyzed BMP6 promoter region and selected three fragments in $\mathrm{CpG}$ islands, the following primers were designed. The first (to amplify fragment 1 base pairs between -1346 to -870 ) was 5'-aggaagagag GGAGGGTGTGTTTTAATTTTTAGTA-3' and 3'-cagta atacgactcactatagggagaaggctACTCAAATACCTCACTCCAA CCC-5'. The second (to amplify fragment 2 base pairs between -892 to -498) was 5'-aggaagagagGGGTTGGAGTGAGGTAT TTGAGT-3' and 3'-cagtaatacgactcactatagggagaaggctCAA AACCACCATAAAATTTACCCC -5'. The third (to amplify fragment 3 base pairs between -521 to -177 ) was 5'-aggaagagag GGGGTAAATTTTATGGTGGTTTT-3' and 3'-cagtaatacg actcactatagggagaaggctCCCAATTACCCAAACTAAAAAAA TAA-5'. The spectra methylation ratios were generated by Epityper software version 1.0 (Sequenom).

Transwell migration assay. Cell migration was assessed using 24-well inserts (Corning Inc., Corning, NY, USA) with $8-\mu \mathrm{m}$ pores according to the manufacturer's protocol. Briefly, cells $\left(1.0 \times 10^{5}\right.$ cells $)$ were seeded into the upper chamber of the system. After $72 \mathrm{~h}$ of incubation, the cells in the upper chamber were removed, and the cells which invaded through the Matrigel matrix membrane were stained with Giemsa assay. After slightly washing, the migrating cells were examined by microscopy at a magnification of $x 40$ with a Nikon microscope (ts100-F, Japan).

Statistical analyses. Data are expressed as the mean \pm SD. The paired t-test was applied for statistical analysis by using the SPSS software (version 13.0). Results were considered statistically significant if 2-tailed P-values were $<0.05$.

\section{Results}

DNA methylation suppresses BMP6 expression in primary breast tumors. To determine the epigenetic silencing of the BMP6 gene in primary breast cancer, firstly, we analyzed BMP6 expression in 16 paired breast cancer samples and matched adjacent normal tissues. Real-time PCR showed that $12 / 16(75 \%)$ breast cancer tissues decreased expression of BMP6 compared to paired adjacent normal tissues; there was a significant difference between tumor tissues and the paired adjacent normal tissues (Fig. 1A). Secondly, we detected BMP6 methylation status in primary breast cancer tissues using Sequenom MassARRAY platform. The mean methylation level of BMP6 of breast cancer tissues was significantly higher than paired adjacent normal tissues (Fig. 1B). Fig. 2 shows the methylation level at different $\mathrm{CpG}$ sites of tumor tissues and adjacent normal tissues and 29/51 (56.86\%) CpG sites showed 
A

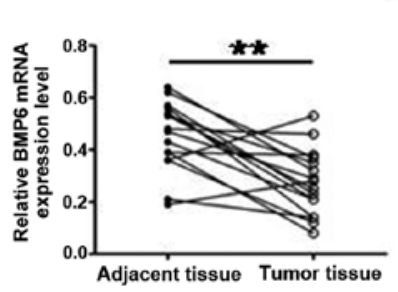

B

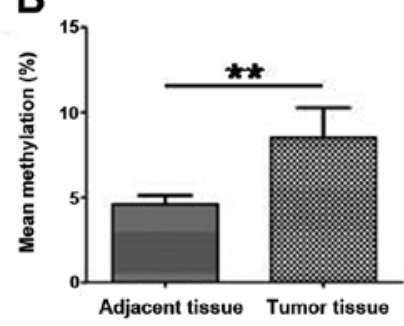

C

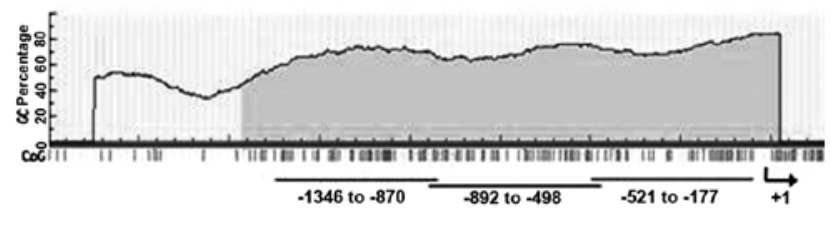

D 5 G'. AACTAAGGAGGGATGGAAAGCTATCATTTTCTTTCTTCCTCAGTTCTGGCAGTATAGTGATTTAATCGCTGTTATTAT ITTATTAGTTAMGCATCACTTAGGCCTTTTAMTGATGGTAMAGAGAMGCTTCAGATCGGGGTATTGGTCAGATCA

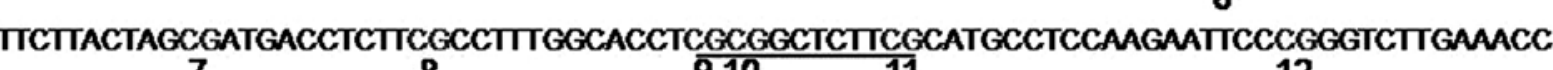
7 8 11 TACGCGCGGCGGAGGCGACGCCTCTCGGGAGAGGGAGGGTGAAGAAGGGCGGGGTATTTAAATAGCCCGCGAGC TTCGCGTCCCCATTGGCTGCCAGCCTGTCGGTTACCGCGGGGCGGGGAGTCCCGGGGCTGGAGTGAGGTACCTGAGC $\begin{array}{lllll}2324 & 25 & 2627 & 28 & 29\end{array}$

E 5 GGGCTGGAGTGAGGTACCTGAGCCGGGCGCGCAGCAGAAAGCCCACGITGGCCGCTGCGGGGAGCGCGGCGTGGAG $\frac{123}{12}$ AGGCGGGAGACCGATTCCGGCGCCGCCGCCGCGGCCGCTGCTCGGTGCACTAGCCCCCTTCCTTCCCATCCTITCTG $10 \quad \begin{array}{lllllll}11 & 12 & 14 & 15 & 1617 & 18 & 19\end{array}$ CGAGCGGGITTGCTGGGCAGCCGGGCGACCGCCCAACGGAAACCAAGATCCTCGСССССTCCGCTTGAGGAGGCGTG $2021 \quad \frac{22}{24} 25 \quad 26 \quad \frac{23}{27} 28 \quad 29 \quad \frac{29}{30}$ CGGCGCGCGGAGATITTGAGTGGGAGCGACGGTGCCCGAGAGCTCAGGGGCGCGGAGTTCGTGAGCGAGAGGAAG

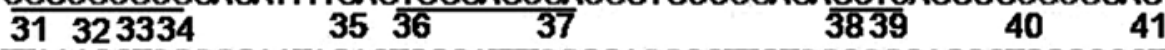
TIAACCTCGCGGAATAGACTGGCATTTCGGACGCCTTGTCGCCGCAGCCTGGGCCCTGCAACGGGGTAACTTCAT GGTGGCCCTG 4243 $\begin{array}{llll}44 & 45 & 46 \quad 47\end{array}$ 48
$F$ G' GGGTAAACTTCATGGTGGCCCTCCO GAGCGGCCCCGCTCCCCGCTECCCCGGGTCCCACTCAGTCGCCAGGGAGAG GCGAGGCCCAGAGTGACCGCGCCGCGACTCGCAGGAGCCAGGGCCAGGCTGCAGIGCAGCCCGGACTCAGACGCAC $1 8 \quad \longdiv { 1 9 2 0 2 1 2 2 } 2 3 \quad 2 4$ CTGGCCTGGACCGCGCGCCTCTAGAGACCTGCGCGAGGCTGTGAGGCTCCCCTTCCTCCCCTCCAAGCGGTCTCCTGG $\overline{272829} \overline{3031}$ TGATCGCCCCTICGCCACCTCTCTAGCCTGGGCAACTGGG 32 33

Figure 1. Real-time PCR and Sequenom MassARRAY analysis of the mRNA expression and promoter DNA methylation level of BMP6 in breast cancer tissues and adjacent normal tissues. (A) Real-time PCR analysis showed that BMP6 mRNA expression was significantly decreased in tumor tissues compared to the paired adjacent tissues. (B) Quantitative methylation analysis showed that DNA methylation level of BMP6 promoter was significantly increased in tumor tissues compared to the paired adjacent tissues. (C) Schematic diagram of the BMP6 promoter and the fragment for the methylation analysis. (D-F) Details of the BMP6 promoter region sequence: (D) base pairs between -1346 to -870 ; (E) base pairs between -892 to -498 ; (F) base pairs between -521 to -177 ; ${ }^{* *} \mathrm{P}<0.01$.

a higher methylation level in tumor tissues compared to the paired adjacent normal tissues.

DNA hypermethylation of BMP6 is related to breast cancer cell drug resistance. In our previous study (16), we found that BMP6 expression was downregulated in the multidrug-resistant breast cancer cell line MCF-7/ADR compared to its parental MCF-7 cells and knockdown of the expression of BMP6 in $\mathrm{MCF}-7$ cells enhanced the chemoresistance to doxorubicin. Here, we found that downregulation of BMP6 in MCF-7/ADR was reversed by treatment of DNA methyltransferase inhibitor 5-aza-dC (Fig. 3A and B). This indicated that downregulation of BMP6 in the drug-resistant cell line MCF-7/ADR might be involved in epigenetic mechanisms. Subsequently, we examined BMP6 methylation status in MCF-7 and MCF-7/ADR cells. Higher level methylation status was detected in MCF-7/ ADR cells compared to the MCF-7 cells (Fig. 3C). In addition, we found that 20/51 (39.21\%) CpG sites showed a higher methylation level in MCF-7/ADR compared to the MCF-7 cells (Fig. 4). It is of note that the methylation level of $\mathrm{CpG}$ 


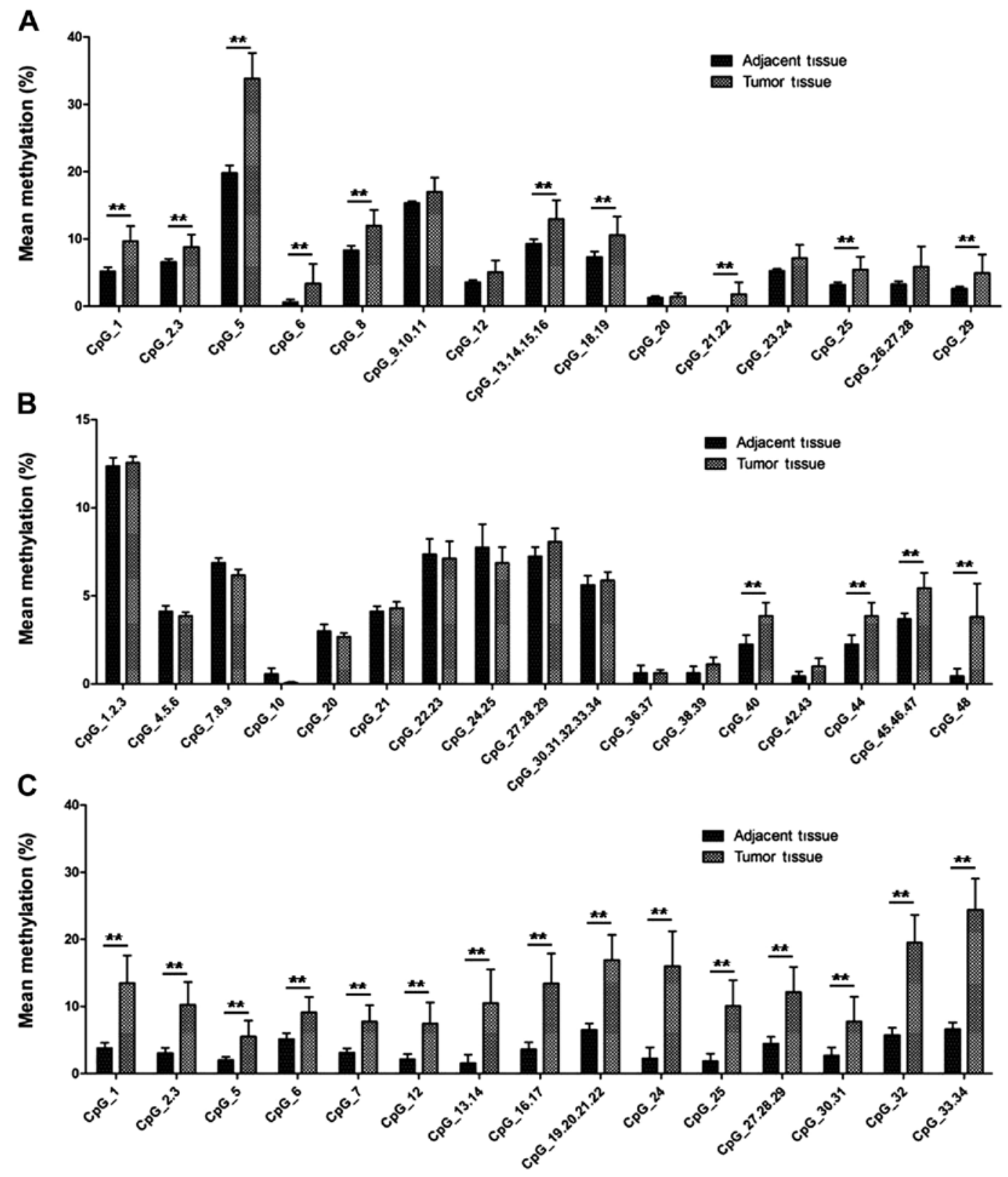

Figure 2. Methylation level of CpG sites in BMP6 gene promoter in tumor tissues and adjacent tissues. (A) Fragment between -1346 to -870; (B) fragment between -892 to -498 ; (C) fragment between -521 to $-177 ;{ }^{* *} \mathrm{P}<0.01$.

A

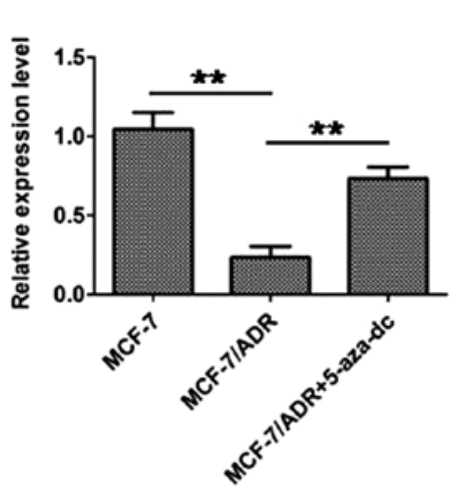

B

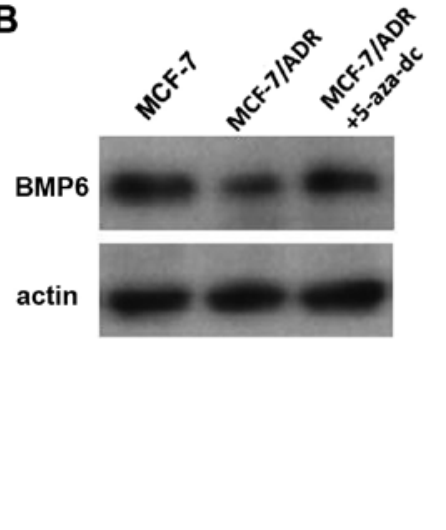

C

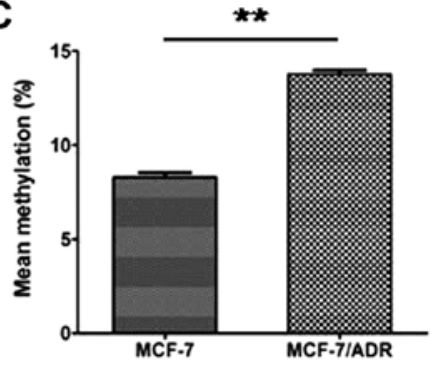

Figure 3. Real-time PCR, western blot and Sequenom MassARRAY analysis of the expression and promoter DNA methylation level of BMP6 in breast cancer cell line MCF-7 and drug-resistant cell line MCF-7/ADR. Real-time PCR (A) and western blotting (B) showed that BMP6 was downregulated in MCF-7/ADR cells and 5-aza-dc treatment reversed the decrease of BMP6 expression. (C) Quantitative methylation analysis showed that DNA methylation level of BMP6 promoter was significantly increased in MCF-7/ADR cells compared to MCF-7 cells; ${ }^{* *} \mathrm{P}<0.01$. 

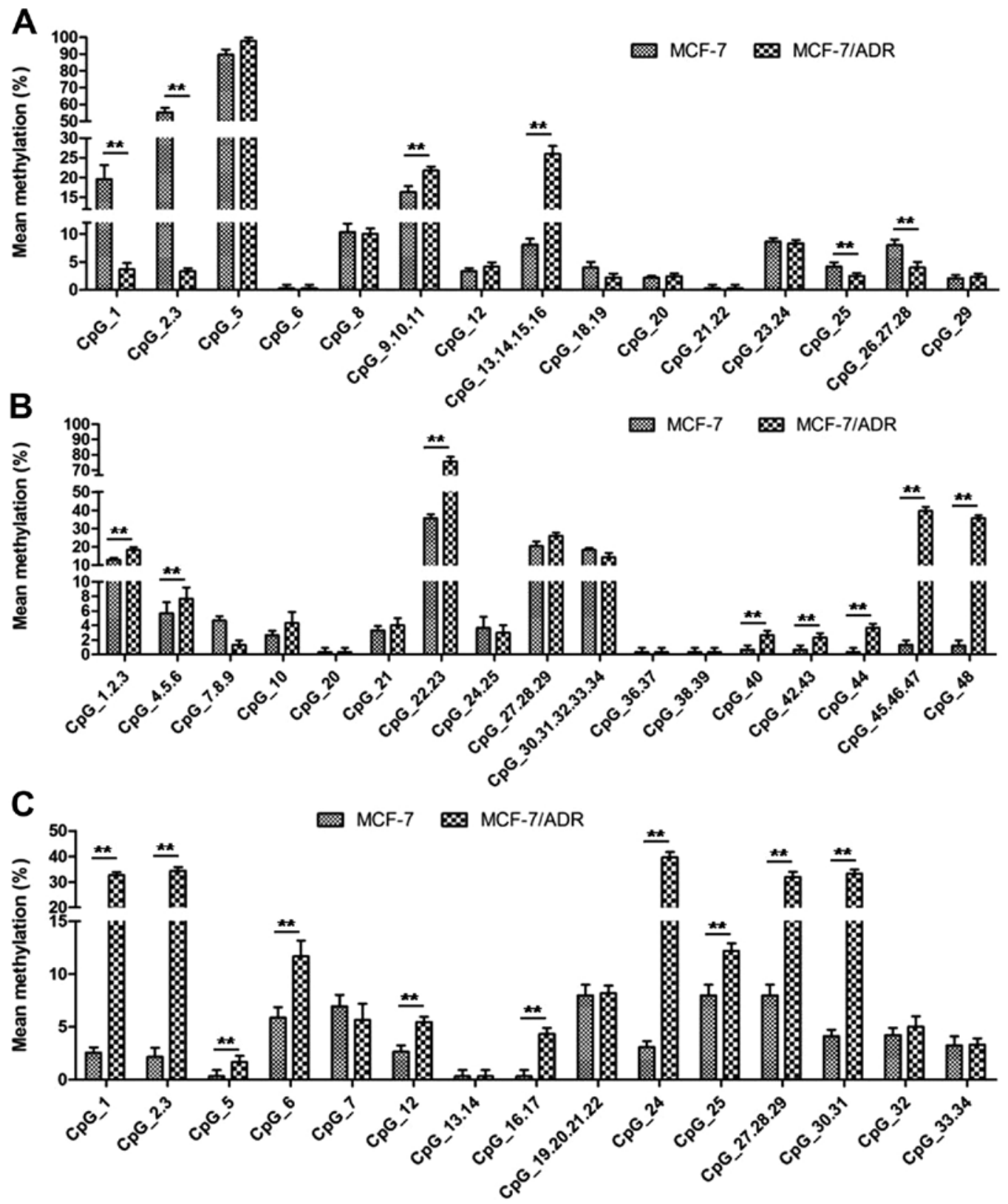

Figure 4. Methylation level of CpG sites in BMP6 gene promoter in MCF-7 and MCF-7/ADR cells. (A) Fragment between -1346 to -870; (B) fragment between -892 to -498 ; (C) fragment between -521 to $-177 ;{ }^{* * *} \mathrm{P}<0.01$.

site 1,2, 3, 25, 26, 27 and 28 in fragment 1 of MCF-7 cells was higher than MCF-7/ADR cells (Fig. 4A).

Downregulation of BMP6 is involved in EMT in MCF-7/ADR cells. In the drug-resistant breast cancer cells MCF-7/ADR, the BMP6 expression was significantly lower than in the MCF-7 cell lines (Fig. 5A and B). In addition, our previous results indicated that downregulation of BMP6 contributed to the breast cancer cell drug resistance. In the present study, low expression BMP6 cells MCF-7/ADR or MCF-7 cells transfected BMP6-specific shRNA showed EMT phenotype. We found that higher BMP6 expression cells (MCF-7 or $\mathrm{MCF}-7 / \mathrm{shNC}$ cells) displayed a cobblestone-like appearance and tight cell-cell junctions (Fig. 5D). In contrast, the lower BMP6 expression cells MCF-7/ADR cells or MCF-7 cells transfected with BMP6-specific shRNA vector) appeared to have spindle-cell morphology; MCF-7/ADR cells treated with BMP6 protein reversed the morphologic changes (Fig. 5D).

Moreover, Transwell assay showed MCF-7/shRNA or MCF-7/ADR cells displayed markedly increased migration ability in comparison with their parental MCF-7 cells and BMP6 recombinant protein treatment significantly inhibited the migration ability of MCF-7/ADR cells (Fig. 5C). Real-time PCR showed that EMT transcription factors such as ZEB1, Snail1 and Twist1 expression were significantly upregulated in MCF-7/ADR or MCF-7 cells transfected with BMP6 specific-shRNA vector (Fig. 5E). The epithelial cell marker E-cadherin expression was significantly downregulated but the mesenchymal marker vimentin was significantly upregulated (Fig. 5E and F). MCF-7/ADR cells treated with BMP6 protein effectively reversed the expression of marker of EMT (Fig. 5E and F). 

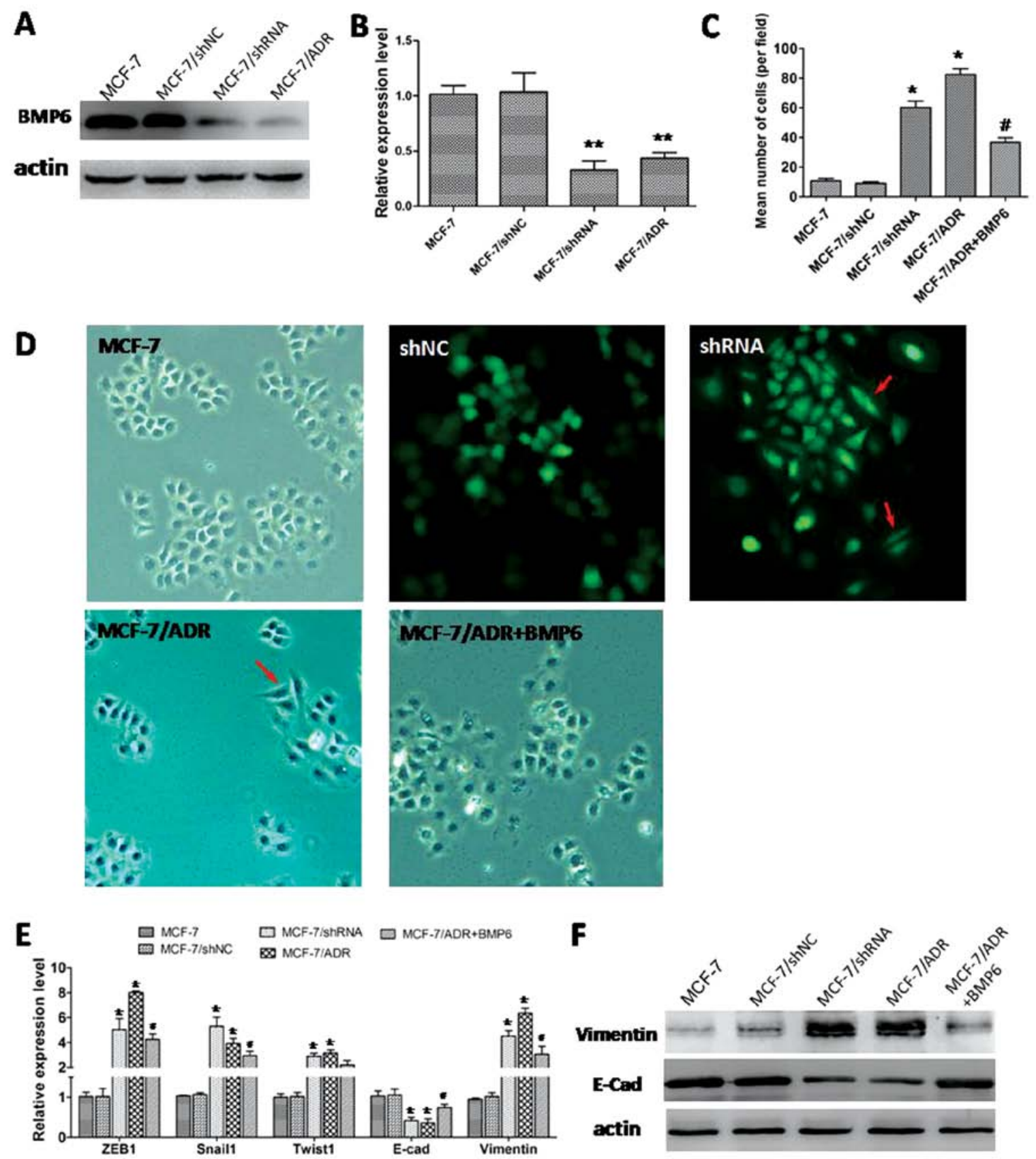

$\mathbf{F}$

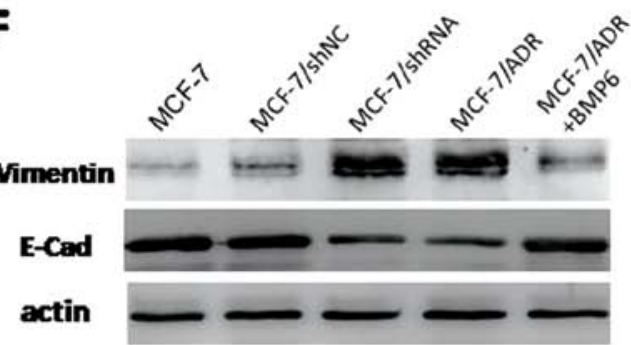

Figure 5. BMP6 expression contributes to the regulation of molecular markers of EMT in breast cancer cells. Western blotting (A) and real-time PCR (B) showed that BMP6 expression was downregulated in the drug-resistant breast cancer cell line MCF-7/ADR or MCF-7 cells transfected with BMP6-specific shRNA vector. (C) Transwell assay showed MCF-7/shRNA or MCF-7/ADR cells displayed markedly increased migration ability in comparison with their parental MCF-7 cells and BMP6 recombinant protein treatment significantly inhibited the migration ability of MCF-7/ADR cells. (D) The morphological characteristics of the MCF-7/ADR and MCF-7 cells transfected with BMP6-specific shRNA vector. MCF7 or MCF-7 cells transfected with shNC vector displayed a cobblestone-like appearance and tight cell-cell junctions. In contrast, the MCF-7/ADR or MCF-7 cells transfected with BMP6-specific shRNA vector appeared to have spindle-cell morphology; MCF-7/ADR cells treated with BMP6 protein reversed the morphologic changes. (E and F) Real-time PCR and western blotting showed the expression of markers of epithelial and mesenchymal phenotypes in MCF-7 cells transfected with BMP6-specific shRNA vector or the drug-resistant cell line MCF-7/ADR treated with BMP6 protein, respectively, ${ }^{*} \mathrm{P}<0.05$ vs. MCF-7 cells; ${ }^{*} \mathrm{P}<0.05$ vs. MCF-7/ADR cells.

\section{Discussion}

In the present study, we found that DNA methylation can regulate the expression of BMP6 in breast cancer. These findings showed a decreased expression of BMP6 and an increase of methylation level of its promoter in breast cancer tissue compared to the paired adjacent normal tissues. In the breast cancer cell line, doxorubicin-resistant MCF-7/ADR cells showed a higher methylation level of BMP6 promoter compared with their parental MCF-7 cells accompanied by the lower expression of BMP6. Moreover, the cells with lower expression of BMP6, MCF-7/ADR cells or MCF-7 cells transfected with the BMP6-specific shRNA vector, showed a typical EMT phenotype. Collectively, our results indicated that hypermethylation of promoter downregulated the expression of BMP6 and then induced the cells to undergo EMT during the acquisition of drug resistance of breast cancer cells. 
BMP6 is a member of the signaling molecules of the TGF- $\beta$ superfamily that are important regulators of cell proliferation and apoptosis in various types of cells including breast cancer, myeloma and lymphoma (16,20,21). BMP6 transfer signals through ligation of type I and type II serine-threonine kinase receptor, which then propagate the signal downstream by phosphorylating receptor-activated Smad protein (22). Previous studies by us and others showed that BMP6 might act as a suppressor of cancer to inhibit the cancer cell proliferation and metastasis (23). Furthermore, we also confirmed that BMP6 played a critical role in breast cancer cell aberrant proliferation and chemoresistance. With the development of breast cancer cell drug resistance, the expression of BMP6 was markedly decreased. However, the regulation of BMP6 downregulation remains unknown in breast cancer since the promoter sequence of BMP6 was identified as a target for aberrant DNA methylation (24).

Herein, we analyzed the methylation status of BMP6 promoter region in primary breast cancer tissues and breast cancer cell line MCF-7 and the drug-resistant cell line MCF-7/ ADR. We found the level of methylation of BMP6 in cancer tissues was significantly higher than the adjacent normal tissues. In cell lines, the MCF-7 cells showed a lower level of methylation of BMP6 than the MCF-7/ADR cells. These indicated that hypermethylation of BMP6 promoter might be involved in breast tumorigenesis and drug resistance. In fact, previous studies also confirmed that aberrant methylation of BMP6 is involved in various cancers including malignant lymphoma, malignant pleural mesothelioma, T-cell leukemia and multiple myeloma $(20,21,25-27)$. In the present study, we noted that aberrant methylation of BMP6 promoter was involved in breast cancer cell drug resistance. The findings suggest that DNA methylation may be important molecular events in the acquisition of drug resistance in breast cancer and the use of hypomethylation agents may be a novel therapeutic strategy for reversal of drug resistance.

Epithelial-mesenchymal transition (EMT) is an essential process for embryonic development and is implicated in tumor progression, invasion and metastasis (28). Previous studies suggested that EMT phenotype was closely related to chemoresistance in cancer cells. An EMT phenotype has been detected in gemcitabine-resistant pancreatic cancer cells $(29,30)$, gefitinib-resistant non-small cell lung cancer (11), oxaliplatinresistant colorectal cancer cells (31), paclitaxel-resistant ovarian cancer cells (32) and 5-fluorouracil or tamoxifenresistant breast cancer cells $(12,33)$. In our study, we found BMP6 expression inhibition caused an EMT phenotype of MCF-7 cells and resistance to doxorubicin. MCF-7/ADR cells or MCF-7 cells transfected with BMP6-specific shRNA vector displayed elongated, irregular fibroblastoid morphology. In contrast, MCF-7 cells or MCF-7 cells transfected with control shRNA vector had a rounded shape, typical of epithelial cells. These changes in phenotype suggest that MCF-7/ADR cells underwent EMT. Results from real-time PCR and western blotting also confirmed the phenotype of EMT in MCF-7/ ADR cells. When the MCF-7/ADR cells were treated with the BMP6 recombinant protein, the morphologic change and EMT-related gene expression could be reversed. These indicated that BMP6 was an important regulator of EMT during the breast cancer cell acquisition of drug resistance.
In conclusion, our data highlight the importance of DNA methylation modifications in determining the expression of BMP6. In addition, downregulation of BMP6 caused by hypermethylation induced an EMT phenotype subsequently during the breast cancer cell acquisition drug resistance. To the best of our knowledge, this is the first study on BMP6 suppression by DNA methylation that modulates the breast cancer chemosensitivity via EMT. The findings suggest that DNA methylation and EMT may be important molecular events in the acquisition of drug resistance in breast cancer and the use of hypomethylation agents may be a novel therapeutic strategy for the reversal of drug resistance.

\section{References}

1. Kalluri R and Weinberg RA: The basics of epithelial-mesenchymal transition. J Clin Invest 119: 1420-1428, 2009.

2. Polyak K and Weinberg RA: Transitions between epithelial and mesenchymal states: acquisition of malignant and stem cell traits. Nat Rev Cancer 9: 265-273, 2009.

3. Thiery JP: Epithelial-mesenchymal transitions in tumour progression. Nat Rev Cancer 2: 442-454, 2002.

4. Mani SA, Guo W, Liao MJ, et al: The epithelial-mesenchymal transition generates cells with properties of stem cells. Cell 133: 704-715, 2008.

5. Kalluri R and Neilson EG: Epithelial-mesenchymal transition and its implications for fibrosis. J Clin Invest 112: 1776-1784, 2003.

6. Lee J.M, Dedhar S, Kalluri R and Thompson EW: The epithelialmesenchymal transition: new insights in signaling, development, and disease. J Cell Biol 172: 973-981, 2006.

7. Hollier B.G, Evans K and Mani SA: The epithelial-to-mesenchymal transition and cancer stem cells: a coalition against cancer therapies. J Mammary Gland Biol Neoplasia 14: 29-43, 2009.

8. Iwatsuki M,Mimori K, Yokobori T, et al: Epithelial-mesenchymal transition in cancer development and its clinical significance. Cancer Sci 101: 293-299, 2010.

9. Frederick BA, Helfrich BA, Coldren CD, Zheng D, Chan D, Bunn PA Jr and Raben D: Epithelial to mesenchymal transition predicts gefitinib resistance in cell lines of head and neck squamous cellcarcinoma and non-small cell lung carcinoma. Mol Cancer Ther 6: 1683-1691, 2007.

10. Uramoto $H$, Iwata $T$, Onitsuka $T$, Shimokawa $H$, Hanagiri $T$ and Oyama T: Epithelial-mesenchymal transition in EGFR-TKI acquired resistant lung adenocarcinoma. Anticancer Res 30: 2513-2517, 2010.

11. Rho JK, Choi YJ, Lee JK, et al: Epithelial to mesenchymal transition derived from repeated exposure to gefitinib determines the sensitivity to EGFR inhibitors in A549, a non-small cell lung cancer cell line. Lung Cancer 63: 219-226, 2009.

12. Zhang W, Feng M, Zheng G, et al: Chemoresistance to 5-fluorouracil induces epithelial-mesenchymal transition via up-regulation of Snail in MCF7 human breast cancer cells. Biochem Biophys Res Commun 417: 679-685, 2012.

13. Haslehurst AM, Koti M, Dharsee M, et al: EMT transcription factors snail and slug directly contribute to cisplatin resistance in ovarian cancer. BMC Cancer 12: 91, 2012.

14. Fojo T: Multiple paths to a drug resistance phenotype: mutations, translocations, deletions and amplification of coding genes or promoter regions, epigenetic changes and microRNAs. Drug Resist Updat 10: 59-67, 2007.

15. Chen GQ, Zhao ZW, Zhou HY, Liu YJ and Yang HJ: Systematic analysis of microRNA involved in resistance of the MCF-7 human breast cancer cell to doxorubicin. Med Oncol 27: 406-415, 2010.

16. Lian WJ, Liu G, Liu YJ, Zhao ZW, Yi T and Zhou HY: Downregulation of BMP6 enhances cell proliferation and chemoresistance via activation of the ERK signaling pathway in breast cancer. Oncol Rep 30: 193-200, 2013.

17. Wang Y and Shang Y: Epigenetic control of epithelial-tomesenchymal transition and cancer metastasis. Exp Cell Res 319: 160-169, 2013.

18. Kiesslich T, Pichler M and Neureiter D: Epigenetic control of epithelial-mesenchymal-transition in human cancer (Review). Mol Clin Oncol 1: 3-11, 2013. 
19. Livak KJ and Schmittgen TD: Analysis of relative gene expression data using real-time quantitative PCR and the 2(-Delta Delta C(T)) method. Methods 25: 402-408, 2001.

20. Daibata M,Nemoto Y, Bandobashi K, et al: Promoter hypermethylation of the bone morphogenetic protein- 6 gene in malignant lymphoma. Clin Cancer Res 13: 3528-3535, 2007.

21. Hashida Y, Nemoto Y, Imajoh M, et al: Promoter methylation of the bone morphogenetic protein 6 gene in multiple myeloma. Oncol Rep 27: 825-830, 2012.

22. Miyazono K, Maeda S and Imamura T: BMP receptor signaling: transcriptional targets, regulation of signals, and signaling crosstalk. Cytokine Growth Factor Rev 16: 251-263, 2005.

23. Kersten C, Dosen G, Myklebust JH, Sivertsen EA, Hystad ME, Smeland EB and Rian E: BMP-6 inhibits human bone marrow B lymphopoiesis - upregulation of Id1 and Id3. Exp Hematol 34 72-81, 2006

24. Tamada H, Kitazawa R, Gohji K, Kamidono S, Maeda S and Kitazawa S: Molecular cloning and analysis of the 5'-flanking region of the human bone morphogenetic protein-6 (BMP-6) Biochim Biophys Acta 1395: 247-251, 1998.

25. Barekati Z, Radpour R, Lu Q, et al: Methylation signature of lymph node metastases in breast cancer patients. BMC Cancer 12: 244,2012

26. Taniguchi A, Nemoto Y, Yokoyama A, Kotani N, Imai S, Shuin T and Daibata M: Promoter methylation of the bone morphogenetic protein- 6 gene in association with adult T-cell leukemia. Int $\mathrm{J}$ Cancer 123: 1824-1831, 2008.
27. Kimura K, Toyooka S, Tsukuda K, et al: The aberrant promoter methylation of BMP3b and BMP6 in malignant pleural mesotheliomas. Oncol Rep 20: 1265-1268, 2008.

28. De Craene B and Berx G: Regulatory networks defining EMT during cancer initiation and progression. Nat Rev Cancer 13: 97-110, 2013.

29. Shah AN, Summy JM, Zhang J, Park SI, Parikh NU and Gallick GE: Development and characterization of gemcitabineresistant pancreatic tumor cells. Ann Surg Oncol 14: 3629-3637, 2007.

30. Wang Z,Li Y, Kong D, et al: Acquisition of epithelial-mesenchymal transition phenotype of gemcitabine-resistant pancreatic cancer cells is linked with activation of the notch signaling pathway. Cancer Res. 69: 2400-2407, 2009.

31. Yang AD, Fan F, Camp ER, et al: Chronic oxaliplatin resistance induces epithelial-to-mesenchymal transition in colorectal cancer cell lines. Clin Cancer Res 12: 4147-4153, 2006.

32. Kajiyama H, Shibata K, Terauchi M, Yamashita M, Ino K, Nawa A and Kikkawa F: Chemoresistance to paclitaxel induces epithelial-mesenchymal transition and enhances metastatic potential for epithelial ovarian carcinoma cells. Int J Oncol 31: 277-283, 2007.

33. Hiscox S, Jiang WG, Obermeier K, et al: Tamoxifen resistance in MCF7 cells promotes EMT-like behaviour and involves modulation of beta-catenin phosphorylation. Int J Cancer 118: 290-301, 2006. 